\title{
Counting descents, rises, and levels, with prescribed first element, in words
}

\author{
Sergey Kitaev $\|^{\prime}$ and Toufik Mansour ${ }^{2}$ and Jeff Remmel ${ }^{3 \mid}$ \\ ${ }^{1}$ The Mathematics Institute, Reykjavik University, IS-103 Reykjavik, Iceland \\ ${ }^{2}$ Department of Mathematics, University of Haifa, 31905 Haifa, Israel \\ ${ }^{3}$ Department of Mathematics, University of California, San Diego, La Jolla, CA 92093, USA
}

received 30 May 2007, revised 4 July 2008, accepted 11 July 2008.

Recently, Kitaev and Remmel refined the well-known permutation statistic "descent" by fixing parity of one of the descent's numbers which was extended and generalized in several ways in the literature. In this paper, we shall fix a set partition of the natural numbers $\mathbb{N},\left(\mathbb{N}_{1}, \ldots, \mathbb{N}_{s}\right)$, and we study the distribution of descents, levels, and rises according to whether the first letter of the descent, rise, or level lies in $\mathbb{N}_{i}$ over the set of words over the alphabet $[k]=\{1, \ldots, k\}$. In particular, we refine and generalize some of the results by Burstein and Mansour.

Keywords: descent, level, rise, set partition, word, composition, multivariate generating function

\section{Introduction}

The descent set of a permutation $\pi=\pi_{1} \cdots \pi_{n} \in S_{n}$ is the set of indices $i$ for which $\pi_{i}>\pi_{i+1}$. This statistic was first studied by MacMahon [13] almost a hundred years ago and it still plays an important role in the field of permutation statistics. The number of permutations of length $n$ with exactly $m$ descents is counted by the Eulerian number $A_{m}(n)$. The Eulerian numbers are the coefficients of the Eulerian polynomials $A_{n}(t)=\sum_{\pi \in S_{n}} t^{1+\operatorname{des}(\pi)}$. It is well-known that the Eulerian polynomials satisfy the identity $\sum_{m \geq 0} m^{n} t^{m}=\frac{A_{n}(t)}{(1-t)^{n+1}}$. For more properties of the Eulerian polynomials see [5].

Recently, Kitaev and Remmel [9] studied the distribution of a refined "descent" statistic on the set of permutations by fixing parity of (exactly) one of the descent's numbers. For example, they showed that the number of permutations in $S_{2 n}$ (resp. $S_{2 n+1}$ ) with exactly $k$ descents such that the first entry of the descent is an even number is given by $\left(\begin{array}{l}n \\ k\end{array}\right)^{2} n !^{2}$ (resp. $\left.\frac{1}{k+1}\left(\begin{array}{l}n \\ k\end{array}\right)^{2}(n+1) !^{2}\right)$. In [10], the authors generalized results of [9] by studying descents according to whether the first or the second element in a descent pair is congruent to $0 \bmod k \geq 2$.

\footnotetext{
${ }^{\dagger}$ The work presented here was supported by grant no. 060005012/3 from the Icelandic Research Fund.

¥The work presented here was partially supported by NSF grant DMS 0654060 
Subsequently, Hall and Remmel [7] generalized results of [10] by considering " $X, Y$-descents," which are descents whose "top" (first element) is in $X$ and whose "bottom" (second element) is in $Y$ where $X$ and $Y$ are any subsets of the natural numbers $\mathbb{N}$. In particular, Hall and Remmel [7] showed that one can reduce the problem of counting the number of permutations $\sigma$ with $k X, Y$-descents to the problem of computing the $k$-th hit number of a Ferrers board in many cases. Liese [11] also considered the situation of fixing equivalence classes of both descent numbers simultaneously. Also, papers [6] and [12] discuss $q$-analogues of some of the results in [7, 9, 10, 11].

Hall and Remmel [7] extended their results on counting permutations with a given number of $X, Y$ descents to words. That is, let $R(\rho)$ be the rearrangement class of the word $1^{\rho_{1}} 2^{\rho_{2}} \cdots m^{\rho_{m}}$ (i.e., $\rho_{1}$ copies of $1, \rho_{2}$ copies of 2 , etc.) where $\rho_{1}+\cdots+\rho_{m}=n$. For any set $X \subseteq \mathbb{N}$ and any set $[m]=\{1,2, \ldots, m\}$, we let $X_{m}=X \cap[m]$ and $X_{m}^{c}=[m]-X$. Then given $X, Y \subseteq \mathbb{N}$ and a word $w=w_{1} \cdots w_{n} \in R(\rho)$, define

$$
\begin{aligned}
\operatorname{Des}_{X, Y}(w) & =\left\{i: w_{i}>w_{i+1} \& w_{i} \in X \& w_{i+1} \in Y\right\} \\
\operatorname{des}_{X, Y}(w) & =\left|D e s_{X, Y}(w)\right|, \text { and } \\
P_{\rho, s}^{X, Y} & =\left|\left\{w \in R(\rho): \operatorname{des}_{X, Y}(w)=s\right\}\right| .
\end{aligned}
$$

Hall and Remmel [7] proved the following theorem by purely combinatorial means.

Theorem 1.1

$$
P_{\rho, s}^{X, Y}=\left(\begin{array}{c}
a \\
\rho_{v_{1}}, \rho_{v_{2}}, \ldots, \rho_{v_{b}}
\end{array}\right) \sum_{r=0}^{s}(-1)^{s-r}\left(\begin{array}{c}
a+r \\
r
\end{array}\right)\left(\begin{array}{c}
n+1 \\
s-r
\end{array}\right) \prod_{x \in X}\left(\begin{array}{c}
\rho_{x}+r+\alpha_{X, \rho, x}+\beta_{Y, \rho, x} \\
\rho_{x}
\end{array}\right),
$$

where $X_{m}^{c}=\left\{v_{1}, v_{2}, \ldots, v_{b}\right\}, a=\sum_{i=1}^{b} \rho_{v_{i}}$, and for any $x \in X_{m}$,

$$
\begin{aligned}
\alpha_{X, \rho, x} & =\sum_{\substack{z \notin X \\
x<z \leq m}} \rho_{z}, \text { and } \\
\beta_{Y, \rho, x} & =\sum_{\substack{z \notin Y \\
1 \leq z<x}} \rho_{z} .
\end{aligned}
$$

In this paper, we shall study similar statistics over the set $[k]^{n}$ of $n$-letter words over a fixed finite alphabet $[k]=\{1,2, \ldots, k\}$. In what follows, $E=\{2,4,6, \ldots\}$ and $O=\{1,3,5, \ldots\}$ are the sets of even and odd numbers respectively. Also, we let $\mathbf{x}[t]=\left(x_{1}, \ldots, x_{t}\right)$. Then given a word $\pi=\pi_{1} \pi_{2} \ldots \pi_{n} \in[k]^{n}$ and a set $X \subseteq \mathbb{N}$, we define the following statistics:

- $\overleftarrow{\operatorname{Des}}_{X}(\pi)=\left\{i: \pi_{i}>\pi_{i+1}\right.$ and $\left.\pi_{i} \in X\right\}$ and $\overleftarrow{\operatorname{des}}_{X}(\pi)=\left|\overleftarrow{\operatorname{Des}}_{X}(\pi)\right|$,

- $\overleftarrow{\operatorname{Ris}}_{X}(\pi)=\left\{i: \pi_{i}<\pi_{i+1}\right.$ and $\left.\pi_{i} \in X\right\}$ and $\overleftarrow{\operatorname{ris}}_{X}(\pi)=\left|\overleftarrow{\operatorname{Ris}}_{X}(\pi)\right|$

- $\operatorname{Lev}_{X}(\pi)=\left\{i: \pi_{i}=\pi_{i+1}\right.$ and $\left.\pi_{i} \in X\right\}$ and $\operatorname{lev}_{X}(\pi)=\left|\operatorname{Lev}_{X}(\pi)\right|$. 
Let $\left(\mathbb{N}_{1}, \ldots, \mathbb{N}_{s}\right)$ be a set partition of the natural numbers $\mathbb{N}$, i.e. $\mathbb{N}=\mathbb{N}_{1} \cup \mathbb{N}_{2} \cup \ldots \cup \mathbb{N}_{s}, \mathbb{N}_{i} \neq \emptyset$ for all $i$, and $\mathbb{N}_{i} \cap \mathbb{N}_{j}=\emptyset$ for $i \neq j$. Then the main goal of this paper is to study the following multivariate generating function (MGF)

$$
A_{k}=A_{k}(\mathbf{x}[s] ; \mathbf{y}[s] ; \mathbf{z}[s] ; \mathbf{q}[s])=\sum_{\pi} \prod_{i=1}^{s} x_{i}^{\overleftarrow{\operatorname{des}_{\mathbb{N}_{i}}}(\pi)} y_{i}^{\overleftarrow{\operatorname{ris}_{\mathbb{N}_{i}}}(\pi)} z_{i}^{\operatorname{lev}_{\mathbb{N}_{i}}(\pi)} q_{i}^{i(\pi)}
$$

where $i(\pi)$ is the number of letters from $\mathbb{N}_{i}$ in $\pi$ and the sum is over all words over $[k]$.

The outline of this paper is as follows. In Section 2 we shall develop some general methods to compute (1.2). In Section 3, we shall concentrate on computing generating functions for the distribution of the number of levels. That is, we shall study $A_{k}$ where set $x_{i}=y_{i}=1$ for all $i$. In Section 4, we shall find formulas for the number of words in $[k]^{n}$ that have $s$ descents that start with an element less than or equal to $t$ (greater than $t$ ) for any $t \leq k$. Note that if we replace a word $w=w_{1} \cdots w_{n} \in[k]^{n}$ by its complement $w^{c}=\left(k+1-w_{1}\right) \cdots\left(k+1-w_{n}\right)$, then it is easy to see that $\overleftarrow{\operatorname{des}}_{[t]}(w)=\overleftarrow{\text { is }}_{\{k+1-t, \ldots, k\}}(w)$ and $\overleftarrow{\operatorname{des}}_{\{t+1, \ldots, k\}}(w)=\overleftarrow{\operatorname{ris}}_{[k-t]}(w)$. Thus we will also obtain formulas for the number of words in $[k]^{n}$ that have $s$ rises that start with an element less than or equal to $t$ (greater than $t$ ) for any $t \leq k$. In Section 5 , we shall apply our results to study the problem of counting the number of words in $[k]^{n}$ with $p$ descents (rises) that start with an element which is congruent to $i \bmod s$ for any $s \geq 2$ and $i=1, \ldots, s$. In particular, if $s \geq 2$ and $\left(\mathbb{N}_{1}, \ldots, \mathbb{N}_{s}\right)$ is the set partition of $\mathbb{N}$ where $\mathbb{N}_{i}=\{x \in \mathbb{N}: x \equiv i \bmod s\}$ for $i=1, \ldots, s$, then we shall study the generating functions

$$
A_{k}^{(s)}(\mathbf{x}[s] ; \mathbf{y}[s] ; \mathbf{z}[s] ; \mathbf{q}[s])=\sum_{\pi} \prod_{i=1}^{s} x_{i}^{\overleftarrow{\operatorname{des}_{N_{i}}}(\pi)} y_{i}^{\overleftarrow{\operatorname{ris}_{\mathbb{N}_{i}}}(\pi)} z_{i}^{{\operatorname{lev\mathbb {N}_{i}}}_{i}(\pi)} q_{i}^{i(\pi)}
$$

and

$$
A_{k}^{(s)}(\mathbf{x}[s] ; \mathbf{y}[s] ; \mathbf{z}[s] ; q)=\sum_{\pi} q^{|\pi|} \prod_{i=1}^{s} x_{i}^{\overleftarrow{\operatorname{des}_{\mathbb{N}_{i}}}(\pi)} y_{i}^{\overleftarrow{\operatorname{ris}_{\mathbb{N}_{i}}(\pi)} z_{i} \operatorname{lev}_{\mathbb{N}_{i}}(\pi)}
$$

Our general results in Section 2 allow us to derive an explicit formula for $A_{k}^{(s)}(\mathbf{x}[s] ; \mathbf{y}[s] ; \mathbf{z}[s] ; \mathbf{q}[s])$ depending on the equivalence class of $k \bmod s$.

For example, in the case where $s=2$, our general result implies that

$$
\begin{aligned}
A_{2 k}^{(2)}\left(q_{1}, q_{2}\right) & =A_{k}\left(x_{1}, x_{2}, y_{1}, y_{2}, z_{1}, z_{2}, q_{1}, q_{2}\right)= \\
& =\sum_{\pi} x_{1}^{\overleftarrow{\operatorname{des}} O(\pi)} x_{2}^{\overleftarrow{\operatorname{des}_{E}}(\pi)} y_{1}^{\overleftarrow{\operatorname{ris}_{O}}(\pi)} y_{2}^{\check{\operatorname{ris}}}(\pi) z_{1}^{\operatorname{lev}_{O}(\pi)} z_{2}^{\operatorname{lev}_{E}(\pi)} q_{1}^{\operatorname{odd}(\pi)} q_{2}^{\operatorname{even}(\pi)} \\
& =\frac{1+\left(\lambda_{1} \mu_{2}+\lambda_{2}\right) \frac{1-\mu_{1}^{k} \mu_{2}^{k}}{1-\mu_{1} \mu_{2}}}{1-\left(\nu_{1} \mu_{2}+\nu_{2}\right) \frac{1-\mu_{1}^{k} \mu_{2}^{k}}{1-\mu_{1} \mu_{2}}}
\end{aligned}
$$

where the sum is over all words over $[2 k]$, even $(\pi)(\operatorname{resp}$. odd $(\pi))$ is the number of even (resp. odd) numbers in $\pi, \lambda_{j}=\frac{q_{j}\left(1-y_{j}\right)}{1-q_{j}\left(z_{j}-y_{j}\right)}, \mu_{i}=\frac{q_{i}\left(z_{i}-x_{i}\right)}{1-q_{i}\left(z_{i}-y_{i}\right)}$, and $\nu_{j}=\frac{q_{j} y_{j}}{1-q_{j}\left(z_{j}-y_{j}\right)}$ for $j=1,2$. Then by specializing the variables appropriately, we will find explicit formulas for the number of words $w \in[2 k]^{n}$ 
such that $\overleftarrow{\operatorname{des}}_{E}(\pi)=p, \overleftarrow{\operatorname{des}}_{O}(\pi)=p, \overleftarrow{\operatorname{ris}}_{E}(\pi)=p, \overleftarrow{\operatorname{ris}}_{O}(\pi)=p$, etc. For example, we prove that the number of $n$-letter words $\pi$ on $[2 k]$ having $\overleftarrow{\operatorname{des}}_{O}(\pi)=p$ (resp. $\overleftarrow{\text { ris }}_{E}(\pi)=p$ ) is given by

$$
\sum_{j=0}^{n-p} \sum_{i=0}^{j}(-1)^{n+p+i} 2^{j}\left(\begin{array}{c}
j \\
k i
\end{array}\right)\left(\begin{array}{c}
i \\
n
\end{array}\right)\left(\begin{array}{c}
n-j \\
p
\end{array}\right) .
$$

In fact, we shall show that similar formulas hold for the number of words $\pi \in[k]^{n}$ with $p$ descents (rises, levels) whose first element is congruent to $t \bmod s$ for any $s \geq 2$ and $0 \leq t \leq s-1$. Our results refine and generalize the results in [4] related to the distribution of descents, levels, and rises in words. Finally, in Section 6, we shall discuss some open questions and further research.

\section{The general case}

We need the following notation:

$$
A_{k}\left(i_{1}, \ldots, i_{m}\right)=A_{k}(\mathbf{x}[t] ; \mathbf{y}[t] ; \mathbf{z}[t] ; \mathbf{q}[t] ; \mathbf{i}[m])=\sum_{\pi} \prod_{i=1}^{t} x_{i}^{\overleftarrow{\operatorname{des}_{N_{i}}}(\pi)} y_{i}^{\overleftarrow{\operatorname{ris}_{\mathbb{S}_{i}}}(\pi)} z_{i}^{\operatorname{lev}_{\mathbb{N}_{i}}(\pi)} q_{i}^{i(\pi)}
$$

where the sum is taken over all words $\pi=\pi_{1} \pi_{2} \cdots$ over $[k]$ such that $\pi_{1} \cdots \pi_{m}=i_{1} \cdots i_{m}$.

From our definitions, we have that

$$
A_{k}=1+\sum_{i=1}^{k} A_{k}(i)
$$

Thus, to find a formula for $A_{k}$, it is sufficient to find a formula for $A_{k}(i)$ for each $i=1,2, \ldots, k$. First let us find a recurrence relation for the generating function $A_{k}(i)$.

Lemma 2.1 For each $s \in \mathbb{N}_{i}, 1 \leq s \leq k$ and $1 \leq i \leq t$, we have

$$
A_{k}(s)=\frac{q_{i} y_{i}}{1-q_{i}\left(z_{i}-y_{i}\right)} A_{k}+\frac{q_{i}\left(1-y_{i}\right)}{1-q_{i}\left(z_{i}-y_{i}\right)}+\frac{q_{i}\left(x_{i}-y_{i}\right)}{1-q_{i}\left(z_{i}-y_{i}\right)} \sum_{j=1}^{s-1} A_{k}(j) .
$$

Proof: From the definitions we have that

$$
\begin{aligned}
A_{k}(s) & =q_{i}+\sum_{j=1}^{k} A_{k}(s, j) \\
& =q_{i}+\sum_{j=1}^{s-1} A_{k}(s, j)+A_{k}(s, s)+\sum_{j=s+1}^{k} A_{k}(s, j) .
\end{aligned}
$$

Let $\pi$ be any $n$-letter word over $[k]$ where $n \geq 2$ and $\pi_{1}=s>\pi_{2}=j$. If we let $\pi^{\prime}=\pi_{2} \pi_{3} \ldots \pi_{n}$, then it is easy to see that

$$
\overleftarrow{\operatorname{des}}_{\mathbb{N}_{i}}(\pi)=1+\overleftarrow{\operatorname{des}}_{\mathbb{N}_{i}}\left(\pi^{\prime}\right), \quad i(\pi)=1+i\left(\pi^{\prime}\right)
$$

It is also easy to see that remaining $4 t-2$ statistics of interest take the same value on $\pi$ and $\pi^{\prime}$. 
This implies that $A_{k}(s, j)=q_{i} x_{i} A_{k}(j)$ for each $1 \leq j<s$. Similarly, $A_{k}(s, s)=q_{i} z_{i} A_{k}(s)$ and $A_{k}(s, j)=q_{i} y_{i} A_{k}(j)$ for $s<j \leq k$. Therefore,

$$
A_{k}(s)=q_{i}+q_{i} x_{i} \sum_{j=1}^{s-1} A_{k}(j)+q_{i} z_{i} A_{k}(s)+q_{i} y_{i} \sum_{j=s+1}^{k} A_{k}(j) .
$$

Using 2.1, we have $\sum_{j=s+1}^{k} A_{k}(j)=A_{k}-\sum_{j=1}^{s-1} A_{k}(j)-A_{k}(s)-1$, and thus

$$
A_{k}(s)=\frac{q_{i} y_{i}}{1-q_{i}\left(z_{i}-y_{i}\right)} A_{k}+\frac{q_{i}\left(1-y_{i}\right)}{1-q_{i}\left(z_{i}-y_{i}\right)}+\frac{q_{i}\left(x_{i}-y_{i}\right)}{1-q_{i}\left(z_{i}-y_{i}\right)} \sum_{j=1}^{s-1} A_{k}(j),
$$

as desired.

Lemma 2.2 For each $k \geq 1$ and $s \in[k]$,

$$
\sum_{j=1}^{s} A_{k}(j)=\sum_{j=1}^{s} \gamma_{j} \prod_{i=j+1}^{s}\left(1-\alpha_{i}\right)
$$

where, for $i \in \mathbb{N}_{m}$ and $i \geq 1, \gamma_{i}=\frac{q_{m} y_{m}}{1-q_{m}\left(z_{m}-y_{m}\right)} A_{k}+\frac{q_{m}\left(1-y_{m}\right)}{1-q_{m}\left(z_{m}-y_{m}\right)}$ and $\alpha_{i}=\frac{q_{m}\left(y_{m}-x_{m}\right)}{1-q_{m}\left(z_{m}-y_{m}\right)}$.

Proof: We proceed by induction on $s$. Note, that given our definitions of $\gamma_{i}$ and $\alpha_{i}$, we can rewrite 2.2 as

$$
A_{k}(s)=\gamma_{s}-\alpha_{s} \sum_{j=1}^{s-1} A_{k}(j)
$$

It follows that

$$
A_{k}(1)=\gamma_{1}
$$

so that 2.3 holds for $s=1$. Thus the base case of our induction holds. Now assume that 2.3 holds for $s$ where $1 \leq s<k$. Then using our induction hypothesis and (2.4), it follows that

$$
\begin{aligned}
& A_{k}(1)+\cdots+A_{k}(s)+A_{k}(s+1) \\
& =\sum_{j=1}^{s} \gamma_{j} \prod_{i=j+1}^{s}\left(1-\alpha_{i}\right)+\gamma_{s+1}-\alpha_{s+1}\left(\sum_{j=1}^{s} \gamma_{j} \prod_{i=j+1}^{s}\left(1-\alpha_{i}\right)\right) \\
& =\gamma_{s+1}-\sum_{j=1}^{s} \gamma_{j} \prod_{i=j+1}^{s+1}\left(1-\alpha_{i}\right) \\
& =\sum_{j=1}^{s+1} \gamma_{j} \prod_{i=j+1}^{s+1}\left(1-\alpha_{i}\right) .
\end{aligned}
$$

Thus the induction step also holds so that 2.3 must hold in general. 
Lemma 2.1 gives that the $A_{k}(i)$ 's, for $1 \leq i \leq k$, are the solution to the following matrix equation

$$
\left(\begin{array}{lllllll}
1 & 0 & 0 & 0 & 0 & \ldots & 0 \\
\alpha_{2} & 1 & 0 & 0 & 0 & \ldots & 0 \\
\alpha_{3} & \alpha_{3} & 1 & 0 & 0 & \ldots & 0 \\
\alpha_{4} & \alpha_{4} & \alpha_{4} & 1 & 0 & \ldots & 0 \\
\vdots & & & \vdots & & & \vdots \\
\alpha_{k} & \alpha_{k} & \alpha_{k} & \alpha_{k} & \alpha_{k} & \ldots & 1
\end{array}\right) \cdot\left(\begin{array}{l}
A_{k}(1) \\
A_{k}(2) \\
\vdots \\
A_{k}(k)
\end{array}\right)=\left(\begin{array}{l}
\gamma_{1} \\
\gamma_{2} \\
\vdots \\
\gamma_{k}
\end{array}\right)
$$

where, for $i \in \mathbb{N}_{m}$ and $i \geq 1, \gamma_{i}=\frac{q_{m} y_{m}}{1-q_{m}\left(z_{m}-y_{m}\right)} A_{k}+\frac{q_{m}\left(1-y_{m}\right)}{1-q_{m}\left(z_{m}-y_{m}\right)}$, and, for $i \in \mathbb{N}_{m}$ and $i \geq 2$, $\alpha_{i}=\frac{q_{m}\left(y_{m}-x_{m}\right)}{1-q_{m}\left(z_{m}-y_{m}\right)}$. Notice that $\alpha_{i}=\alpha_{j}$ and $\gamma_{i}=\gamma_{j}$ whenever $i$ and $j$ are from the same set $\mathbb{N}_{m}$ for some $m$. In fact, it is easy to see that (2.2) and (2.3) imply that

$$
A_{k}(i)=\gamma_{i}-\alpha_{i} \sum_{j=1}^{i-1} \gamma_{j} \prod_{i=j+1}^{i-1}\left(1-\alpha_{i}\right)
$$

holds for $i=1, \ldots, k$ so that 2.5) has an explicit solution. By combining 2.1] and 2.6), we can obtain the following result.

Theorem 2.3 For $\alpha_{i}$ and $\gamma_{i}$ as above (defined in Lemma 2.2), we have

$$
A_{k}=1+\sum_{j=1}^{k} \gamma_{j} \prod_{i=j+1}^{k}\left(1-\alpha_{i}\right)
$$

solving which for $A_{k}$ gives

$$
A_{k}=\frac{1+\sum_{j=1}^{k} \frac{q_{j}\left(1-y_{j}\right)}{1-q_{j}\left(z_{j}-y_{j}\right)} \prod_{i=j+1}^{k} \frac{1-q_{i}\left(z_{i}-x_{i}\right)}{1-q_{i}\left(z_{i}-y_{i}\right)}}{1-\sum_{j=1}^{k} \frac{q_{j} y_{j}}{1-q_{j}\left(z_{j}-y_{j}\right)} \prod_{i=j+1}^{k} \frac{1-q_{i}\left(z_{i}-x_{i}\right)}{1-q_{i}\left(z_{i}-y_{i}\right)}}
$$

where for each variable $a \in\{x, y, z, q\}$ we have $a_{i}=a_{m}$ if $i \in \mathbb{N}_{m}$.

Even though we state Theorem 2.3 as the main theorem in this paper, its statement can be (easily) generalized if one considers compositions instead of words (see [8]). Indeed, let

$$
B_{k}=B_{k}(\mathbf{x}[t] ; \mathbf{y}[t] ; \mathbf{z}[t] ; \mathbf{q}[t] ; v)=\sum_{\pi} v^{|\pi|} \prod_{i=1}^{t} x_{i}^{\overleftarrow{\operatorname{des}_{\mathbb{N}_{i}}}(\pi)} y_{i}^{\overleftarrow{\operatorname{ris}_{\mathbb{N}_{i}}}(\pi)} z_{i}^{\operatorname{lev}_{\mathbb{N}_{i}}(\pi)} q_{i}^{i(\pi)}
$$

where the sum is taken over all compositions $\pi=\pi_{1} \pi_{2} \cdots$ with parts in $[k]$ and $|\pi|=\pi_{1}+\pi_{2}+\cdots$ is the weight of the composition $\pi$. Also, we let

$$
B_{k}\left(i_{1}, \ldots, i_{m}\right)=B_{k}(\mathbf{x}[t] ; \mathbf{y}[t] ; \mathbf{z}[t] ; \mathbf{q}[t] ; \mathbf{i}[m] ; v)=\sum_{\pi} v^{|\pi|} \prod_{i=1}^{t} x_{i}^{\overleftarrow{\operatorname{des}_{\mathbb{N}_{i}}}(\pi)} y_{i}^{\overleftarrow{\operatorname{ris}_{\mathbb{N}_{i}}}(\pi)} z_{i}^{\operatorname{lev}_{\mathbb{N}_{i}}(\pi)} q_{i}^{i(\pi)}
$$

where again the sum is taken over all compositions $\pi=\pi_{1} \pi_{2} \cdots$ with parts in $[k]$. 
Next, one can copy the arguments of Lemma 2.1 substituting $q_{i}$ by $v^{s} q_{i}$ to obtain the following generalization of Lemma 2.1

$$
B_{k}(s)=\frac{v^{s} q_{i} y_{i}}{1-q_{i}\left(z_{i}-y_{i}\right)} B_{k}+\frac{v^{s} q_{i}\left(1-y_{i}\right)}{1-q_{i}\left(z_{i}-y_{i}\right)}+\frac{v^{s} q_{i}\left(x_{i}-y_{i}\right)}{1-q_{i}\left(z_{i}-y_{i}\right)} \sum_{j=1}^{s-1} B_{k}(j) .
$$

One can then prove the obvious analogue of Lemma 2.1 by induction and apply it to prove the following theorem.

Theorem 2.4 We have

$$
B_{k}=1+\sum_{j=1}^{k} \gamma_{j} \prod_{i=j+1}^{k}\left(1-\alpha_{i}\right)
$$

where $\gamma_{i}=\frac{v^{i} q_{m} y_{m}}{1-q_{m}\left(z_{m}-y_{m}\right)} B_{k}+\frac{v^{i} q_{m}\left(1-y_{m}\right)}{1-q_{m}\left(z_{m}-y_{m}\right)}$, and $\alpha_{i}=\frac{v^{i} q_{m}\left(y_{m}-x_{m}\right)}{1-q_{m}\left(z_{m}-y_{m}\right)}$ if $i$ belongs to $\mathbb{N}_{m}$. Thus,

$$
B_{k}=\frac{1+\sum_{j=1}^{k} \frac{v^{j} q_{j}\left(1-y_{j}\right)}{1-q_{j}\left(z_{j}-y_{j}\right)} \prod_{i=j+1}^{k} \frac{1-q_{i}\left(z_{i}-y_{i}+v^{i}\left(y_{i}-x_{i}\right)\right)}{1-q_{i}\left(z_{i}-y_{i}\right)}}{1-\sum_{j=1}^{k} \frac{v^{j} q_{j} y_{j}}{1-q_{j}\left(z_{j}-y_{j}\right)} \prod_{i=j+1}^{k} \frac{1-q_{i}\left(z_{i}-y_{i}+v^{i}\left(y_{i}-x_{i}\right)\right)}{1-q_{i}\left(z_{i}-y_{i}\right)}}
$$

where for each variable $a \in\{x, y, z, q\}$ we have $a_{i}=a_{m}$ if $i \in \mathbb{N}_{m}$.

Theorem 2.4 can be viewed as a $q$-analogue of Theorem 2.3. (Set $v=1$ in Theorem 2.4 to get Theorem 2.3.)

\section{Counting words by the types of levels}

Suppose we are given a set partition $\mathbb{N}=\mathbb{N}_{1} \cup \mathbb{N}_{2} \cup \cdots \cup \mathbb{N}_{s}$. First observe that for any fixed $i$, if we want the distribution of words in $[k]^{n}$ according to the number of levels which involve elements in $\mathbb{N}_{i}$, then it is easy to see by symmetry that the distribution will depend only on the cardinality of $\mathbb{N}_{i} \cap[k]$. Thus we only need to consider the case where $s=2$ and $\mathbb{N}_{1}=\{1, \ldots, t\}$ for some $t \leq k$.

Let

$$
\begin{aligned}
\lambda_{j} & =\frac{q_{j}\left(1-y_{j}\right)}{1-q_{j}\left(z_{j}-y_{j}\right)}, \\
\nu_{j} & =\frac{q_{j} y_{j}}{1-q_{j}\left(z_{j}-y_{j}\right)}, \text { and } \\
\mu_{i} & =\frac{1-q_{i}\left(z_{i}-x_{i}\right)}{1-q_{i}\left(z_{i}-y_{i}\right)} .
\end{aligned}
$$

Then we can rewrite 2.77 for any arbitrary set partition $\mathbb{N}=\mathbb{N}_{1} \cup \mathbb{N}_{2} \cup \cdots \cup \mathbb{N}_{s}$ as

$$
A_{k}=\frac{1+\sum_{j=1}^{k} \lambda_{j} \prod_{i=j+1}^{k} \mu_{i}}{1-\sum_{j=1}^{k} \nu_{j} \prod_{i=j+1}^{k} \mu_{i}}
$$

where for each variable $a \in\{x, y, z, q\}$, we have $a_{i}=a_{m}$ if $i \in \mathbb{N}_{m}$. 
Suppose we set $x_{1}=x_{2}=y_{1}=y_{2}=z_{2}=1$ and $q_{1}=q_{2}=q$ in (3.4) in the special case where $s=2$ and $\mathbb{N}_{1}=[t]$ for some $t \leq k$. Then $\lambda_{1}=\lambda_{2}=0, \nu_{1}=\frac{q}{1-q\left(z_{1}-1\right)}, \nu_{2}=q$, and $\mu_{1}=\mu_{2}=1$. It follows in this case that

$$
\begin{aligned}
A_{k} & =\frac{1}{1-\left(\frac{t q}{1-q\left(z_{1}-1\right)}+q(k-t)\right)} \\
& =\sum_{m \geq 0} q^{m}\left(\frac{t}{1-q\left(z_{1}-1\right)}+(k-t)\right)^{m} \\
& =\sum_{m \geq 0} q^{m} \sum_{i=0}^{m}\left(\begin{array}{c}
m \\
i
\end{array}\right)(k-t)^{m-i} t^{i}\left(\frac{1}{1-q\left(z_{1}-1\right)}\right)^{i}
\end{aligned}
$$

Since

$$
\begin{aligned}
\left(\frac{1}{1-q\left(z_{1}-1\right)}\right)^{i} & =\sum_{a \geq 0} \frac{(i)_{a}}{a !} q^{a}\left(z_{1}-1\right)^{a} \\
& =\sum_{a \geq 0}\left(\begin{array}{c}
i+a-1 \\
a
\end{array}\right) q^{a}\left(z_{1}-1\right)^{a}
\end{aligned}
$$

it follows that

$$
A_{k}=\sum_{n \geq 0} q^{n} \sum_{m=0}^{n} \sum_{i=0}^{m}\left(\begin{array}{c}
m \\
i
\end{array}\right)\left(\begin{array}{c}
i+n-m-1 \\
n-m
\end{array}\right)(k-t)^{m-i} t^{i}\left(z_{1}-1\right)^{n-m} .
$$

Thus taking the coefficient of $z_{1}^{s}$ on both sides of (3.6), we obtain the following result.

Theorem 3.1 Let $\mathbb{N}=\mathbb{N}_{1} \cup \mathbb{N}_{2}$ where $\mathbb{N}_{1}=[t]$ and $\mathbb{N}_{2}=\mathbb{N}-\mathbb{N}_{1}$. Then if $t \leq k$, the number of words in $[k]^{n}$ with s levels that start with elements in $\mathbb{N}_{1}$ is

$$
\sum_{m=0}^{n} \sum_{i=0}^{m}(-1)^{n-m-s}\left(\begin{array}{c}
m \\
i
\end{array}\right)\left(\begin{array}{c}
i+n-m-1 \\
n-m
\end{array}\right)\left(\begin{array}{c}
n-m \\
s
\end{array}\right)(k-t)^{m-i} t^{i} .
$$

Going back to the general set partition $\mathbb{N}=\mathbb{N}_{1} \cup \mathbb{N}_{2} \cup \cdots \cup \mathbb{N}_{s}$, we can obtain a general formula for the number of words in $[k]^{n}$ for which there are $t_{i}$ levels which start with an element of $\mathbb{N}_{i}$ for $i=1, \ldots, s$ as follows. Let $n_{i}=\left|\mathbb{N}_{i} \cap[k]\right|$ for $i=1, \ldots, n$. Then if set $x_{j}=y_{j}=1$ and $q_{j}=q$ for all $j$, then it will be the case that $\lambda_{j}=0$ and $\mu_{j}=1$ and $\nu_{j}=\frac{q}{1-q\left(z_{j}-1\right)}$ for all $j$. It easy follows that in this case,

$$
\begin{aligned}
A_{k} & =\frac{1}{1-\left(\sum_{i=1}^{s} \frac{n_{i} q}{1-q\left(z_{i}-1\right)}\right)} \\
& =\sum_{m \geq 0} q^{m}\left(\sum_{i=1}^{s} \frac{n_{i}}{1-q\left(z_{i}-1\right)}\right)^{m} \\
& =\sum_{m \geq 0} q^{m} \sum_{\substack{a_{1}+\cdots a_{s}=m \\
a_{1}, \ldots, a_{s} \geq 0}}\left(\begin{array}{c}
m \\
a_{1}, \ldots, a_{m}
\end{array}\right) \prod_{i=1}^{s}\left(\frac{n_{i}}{1-q\left(z_{i}-1\right)}\right)^{a_{i}} .
\end{aligned}
$$


Then using [3.5], we see that

$$
\begin{aligned}
A_{k} & =\sum_{m \geq 0} q^{m} \sum_{\substack{a_{1}+\cdots+a_{s}=m \\
a_{1}, \ldots, a_{s} \geq 0}}\left(\begin{array}{c}
m \\
a_{1}, \ldots, a_{m}
\end{array}\right) n_{1}^{a_{1}} \cdots n_{s}^{a_{s}} \prod_{i=1}^{s} \sum_{b_{i} \geq 0} \frac{\left(a_{i}\right)_{b_{i}}}{b_{i} !} q^{b_{i}}\left(z_{1}-1\right)^{b_{i}} \\
& \left.=\sum_{n \geq 0} q^{n} \sum_{\substack{n \\
m=0}} \sum_{\substack{a_{1}+\ldots+a_{s}=m \\
a_{1}, \ldots, a_{s} \geq 0 \\
b_{1}+\cdots+b_{s}=n-m \\
b_{1}, \ldots, b_{s} \geq 0}}\left(\begin{array}{c}
m \\
a_{1}, \ldots, a_{m}
\end{array}\right) n_{1}^{a_{1}} \cdots n_{s}^{a_{s}} \prod_{i=1}^{s}\left(\begin{array}{c}
a_{i}+b_{i}-1 \\
b_{i}
\end{array}\right)\left(z_{i}-1\right\rangle \mathbf{g}^{\mathbf{s}} \mathrm{i} 8\right)
\end{aligned}
$$

Taking the coefficient of $z_{1}^{t_{1}} \cdots z_{s}^{t_{s}}$ on both sides of 3.8, we obtain the following result.

Theorem 3.2 Let $\mathbb{N}=\mathbb{N}_{1} \cup \cdots \cup \mathbb{N}_{s}$ be a set partition of $\mathbb{N}$. Let $n_{i}=\left|\mathbb{N}_{i} \cap[k]\right|$ for $i=1, \ldots$, s. Then the number of words in $[k]^{n}$ with $t_{i}$ levels that start with elements in $\mathbb{N}_{i}$ for $i=1, \ldots, s$ is

$$
\sum_{m=0}^{n} \sum_{\substack{a_{1}+\cdots+a_{s}=m \\
a_{1}, \ldots, a_{s} \geq 0}} \sum_{\substack{b_{1}+\ldots+b_{s}=n-m \\
b_{1}, \ldots, b_{s} \geq 0}}\left(\begin{array}{c}
m \\
a_{1}, \ldots, a_{m}
\end{array}\right) n_{1}^{a_{1}} \cdots n_{s}^{a_{s}} \prod_{i=1}^{s}\left(\begin{array}{c}
a_{i}+b_{i}-1 \\
b_{i}
\end{array}\right)\left(\begin{array}{c}
b_{i} \\
t_{i}
\end{array}\right)
$$

\section{Classifying words by the number of descents that start with el- ements $\leq t(\geq t+1)$.}

In this section, we shall consider the set partition $\mathbb{N}=\mathbb{N}_{1} \cup \mathbb{N}_{2}$ where $\mathbb{N}_{1}=[t]$. Now if $t \leq k$, then it is easy to see that we can rewrite 2.7] as

$$
A_{k}=\frac{1+\sum_{j=1}^{k} \lambda_{j} \prod_{i=j+1}^{k} \mu_{i}}{1-\sum_{j=1}^{k} \nu_{j} \prod_{i=j+1}^{k} \mu_{i}}
$$

where

$$
\lambda_{j}=\frac{q_{1}\left(1-y_{1}\right)}{1-q_{1}\left(z_{1}-y_{1}\right)}, \quad \nu_{j}=\frac{q_{1} y_{1}}{1-q_{1}\left(z_{1}-y_{1}\right)}, \text { and } \mu_{j}=\frac{1-q_{1}\left(z_{1}-x_{1}\right)}{1-q_{1}\left(z_{1}-y_{1}\right)} \text { if } j \leq t
$$

and

$$
\lambda_{j}=\frac{q_{2}\left(1-y_{2}\right)}{1-q_{2}\left(z_{2}-y_{2}\right)}, \quad \nu_{j}=\frac{q_{2} y_{2}}{1-q_{2}\left(z_{2}-y_{2}\right)}, \text { and } \mu_{j}=\frac{1-q_{2}\left(z_{2}-x_{2}\right)}{1-q_{2}\left(z_{2}-y_{2}\right)} \text { if } j>t .
$$

Now if we want to find formulas for the number of words in $[k]^{n}$ with $s$ descents that start with an element less than or equal to $t$, then we need to set $x_{2}=y_{1}=y_{2}=z_{1}=z_{2}=1$ and $q_{1}=q_{2}=q$ in (4.1). In that case, we will have $\lambda_{j}=0$ and $\nu_{j}=q$ for all $j, \mu_{j}=1+q\left(x_{1}-1\right)$ for $j \leq t$, and $\mu_{j}=1$ for $j>t$. It 
follows that

$$
\begin{aligned}
A_{k} & =\frac{1}{1-\left(\sum_{j=t+1}^{k} q+\sum_{j=1}^{t} q \prod_{i=j+1}^{t}\left(1+q\left(x_{1}-1\right)\right)\right)} \\
& =\frac{1}{1-\left((k-t) q+q \frac{\left(1+q\left(x_{1}-1\right)\right)^{t}-1}{\left(1+q\left(x_{1}-1\right)\right)-1}\right)} \\
& =\frac{1}{1-\frac{1}{\left(x_{1}-1\right)}\left((k-t) q\left(x_{1}-1\right)-1+\left(1+q\left(x_{1}-1\right)\right)^{t}\right)} \\
& =\sum_{m \geq 0} \frac{1}{\left(x_{1}-1\right)^{m}}\left((k-t) q\left(x_{1}-1\right)-1+\left(1+q\left(x_{1}-1\right)\right)^{t}\right)^{m} \\
& =\sum_{m \geq 0} \frac{1}{\left(x_{1}-1\right)^{m}} \sum_{a=0}^{m}\left(\begin{array}{c}
m \\
a
\end{array}\right)\left((k-t) q\left(x_{1}-1\right)-1\right)^{m-a}\left(1+q\left(x_{1}-1\right)\right)^{t a} \\
& =\sum_{m \geq 0} \frac{1}{\left(x_{1}-1\right)^{m}} \sum_{a=0}^{m} \sum_{b=0}^{m-a} \sum_{c=0}^{t a}\left(\begin{array}{c}
m \\
a
\end{array}\right)\left(\begin{array}{c}
m-a \\
b
\end{array}\right)\left(\begin{array}{c}
t a \\
c
\end{array}\right)(-1)^{m-a-b} q^{b}(k-t)^{b}\left(x_{1}-1\right)^{b} q^{c}\left(x_{1}-1\right)^{c} .
\end{aligned}
$$

If we want to take the coefficient of $q^{n}$, then we must have $b+c=n$ or $c=n-b$. Thus

$$
A_{k}=\sum_{n \geq 0} q^{n} \sum_{m \geq 0} \sum_{a=0}^{m} \sum_{b=0}^{m-a}\left(\begin{array}{c}
m \\
a
\end{array}\right)\left(\begin{array}{c}
m-a \\
b
\end{array}\right)\left(\begin{array}{c}
t a \\
n-b
\end{array}\right)(-1)^{m-a-b}(k-t)^{b}\left(x_{1}-1\right)^{n-m} .
$$

Taking the coefficient of $q^{n}$ of both sides of 4.2 , we see that

$$
\sum_{\pi \in[k]^{n}} x_{1}^{\overleftarrow{\operatorname{des}}_{[t]}(\pi)}=\sum_{m \geq 0} \sum_{a=0}^{m} \sum_{b=0}^{m-a}\left(\begin{array}{c}
m \\
a
\end{array}\right)\left(\begin{array}{c}
m-a \\
b
\end{array}\right)\left(\begin{array}{c}
t a \\
n-b
\end{array}\right)(-1)^{m-a-b}(k-t)^{b}\left(x_{1}-1\right)^{n-m}
$$

for all $n$. However, if we replace $x_{1}$ by $z+1$ in (4.3), we see that the polynomial

$$
\sum_{\pi \in[k]^{n}}(z+1)^{\overleftarrow{\operatorname{des}}_{[t]}(\pi)}
$$

has the Laurent expansion

$$
\sum_{m \geq 0} \sum_{a=0}^{m} \sum_{b=0}^{m-a}\left(\begin{array}{c}
m \\
a
\end{array}\right)\left(\begin{array}{c}
m-a \\
b
\end{array}\right)\left(\begin{array}{c}
t a \\
n-b
\end{array}\right)(-1)^{m-a-b}(k-t)^{b} z^{n-m} .
$$

It follows that it must be the case that

$$
\sum_{m \geq n+1} \sum_{a=0}^{m} \sum_{b=0}^{m-a}\left(\begin{array}{c}
m \\
a
\end{array}\right)\left(\begin{array}{c}
m-a \\
b
\end{array}\right)\left(\begin{array}{c}
t a \\
n-b
\end{array}\right)(-1)^{m-a-b}(k-t)^{b} z^{n-m}=0,
$$


so that

$$
A_{k}=\sum_{n \geq 0} q^{n} \sum_{m=0}^{n} \sum_{a=0}^{m} \sum_{b=0}^{m-a}(-1)^{m-a-b}\left(\begin{array}{c}
m \\
a
\end{array}\right)\left(\begin{array}{c}
m-a \\
b
\end{array}\right)\left(\begin{array}{c}
t a \\
n-b
\end{array}\right)(k-t)^{b}\left(x_{1}-1\right)^{n-m}
$$

Thus if we take the coefficient of $x_{1}^{s}$ on both sides of (4.4) and we use the remark in the introduction that $\overleftarrow{\operatorname{des}}_{[t]}(w)=\overleftarrow{\operatorname{ris}}_{\{k+1-t, \ldots, k\}}(w)$ for all $w \in[k]^{n}$, then we see that the number of words $w \in[k]^{n}$ such that $\overleftarrow{\operatorname{des}}_{[t]}(w)=s\left(\overleftarrow{\operatorname{ris}}_{\{k+1-t, \ldots, k\}}(w)=s\right)$ is equal to

$$
\sum_{m=0}^{n-s} \sum_{a=0}^{m} \sum_{b=0}^{m-a}(-1)^{n-a-b-s}\left(\begin{array}{c}
m \\
a
\end{array}\right)\left(\begin{array}{c}
m-a \\
b
\end{array}\right)\left(\begin{array}{c}
t a \\
n-b
\end{array}\right)\left(\begin{array}{c}
n-m \\
s
\end{array}\right)(k-t)^{b} .
$$

Reordering the summands, we see that 4.5 is equal to

$$
\sum_{b=0}^{n-s}(k-t)^{b} \sum_{a=b}^{n-s}(-1)^{n-a-b-s}\left(\begin{array}{c}
t a \\
n-b
\end{array}\right) \sum_{m=a+b}^{n-s}\left(\begin{array}{c}
m \\
a
\end{array}\right)\left(\begin{array}{c}
m-a \\
b
\end{array}\right)\left(\begin{array}{c}
n-m \\
s
\end{array}\right) .
$$

However it is the case that

$$
\sum_{m=a+b}^{n-s}\left(\begin{array}{c}
m \\
a
\end{array}\right)\left(\begin{array}{c}
m-a \\
b
\end{array}\right)\left(\begin{array}{c}
n-m \\
s
\end{array}\right)=\left(\begin{array}{c}
n+1 \\
a+b+s+1
\end{array}\right)\left(\begin{array}{c}
a+b \\
a
\end{array}\right)
$$

This is easy to see combinatorially. That is, we can interpret the RHS of 4.7) as the number of ways of choosing $a+b+1+s$ points, $1 \leq x_{1}<x_{2}<\cdots<x_{a+b+1+s} \leq n+1$, from the set $[n+1]$ and then circling $a$ of the points from $x_{1}<\cdots<x_{a+b}$. However if we classify our choices by the value $m+1$ of $x_{a+b+1}$, then we see that $\left(\begin{array}{c}m \\ a\end{array}\right)$ can be viewed as the number of ways to pick the circled points from $[m],\left(\begin{array}{c}m-a \\ b\end{array}\right)$ can be viewed as the number of ways to pick the non-circled chosen points from $[m]$, and the binomial coefficient $\left(\begin{array}{c}n-m \\ s\end{array}\right)$ can be viewed as the number of ways to pick the points $x_{a+b+2}<\cdots<x_{a+b+1+s}$ from the interval $\{m+2, \ldots, n+1\}$.

Thus we have proved the following theorem.

Theorem 4.1 If $t \leq k$, then the number of words $w \in[k]^{n}$ such that $\overleftarrow{\operatorname{des}}_{[t]}(w)=s\left(\overleftarrow{\operatorname{ris}}_{\{k+1-t, \ldots, k\}}(w)=\right.$ s) is equal to

$$
\sum_{b=0}^{n-s}(k-t)^{b} \sum_{a=b}^{n-s}(-1)^{n-a-b-s}\left(\begin{array}{c}
t a \\
n-b
\end{array}\right)\left(\begin{array}{c}
n+1 \\
a+b+s+1
\end{array}\right)\left(\begin{array}{c}
a+b \\
a
\end{array}\right) .
$$

If we want to find formulas for the number of words in $[k]^{n}$ with $s$ descents that start with an element greater than $t$, then we need to set $x_{1}=y_{1}=y_{2}=z_{1}=z_{2}=1$ and $q_{1}=q_{2}=q$ in (4.1). In that case, we will have $\lambda_{j}=0$ and $\nu_{j}=q$ for all $j, \mu_{j}=1+q\left(x_{2}-1\right)$ for $j>t$, and $\mu_{j}=1$ for $j \leq t$. It follows 
that

$$
\begin{aligned}
A_{k} & =\frac{1}{1-\left(\sum_{j=0}^{t} q\left(1+q\left(x_{2}-1\right)\right)^{k-t}+\sum_{j=t+1}^{k} q \prod_{i=j+1}^{k}\left(1+q\left(x_{2}-1\right)\right)\right)} \\
& =\frac{1}{1-\left(q t\left(1+q\left(x_{2}-1\right)\right)^{k-t}+q \frac{\left(1+q\left(x_{2}-1\right)\right)^{k-t}-1}{\left(1+q\left(x_{2}-1\right)\right)-1}\right)} \\
& =\frac{1}{1-\frac{1}{\left(x_{2}-1\right)}\left(q t\left(x_{2}-1\right)\left(1+q\left(x_{2}-1\right)\right)^{k-t}+\left(1+q\left(x_{1}-1\right)\right)^{k-t}-1\right)} \\
& =\frac{1}{1-\frac{1}{\left(x_{2}-1\right)}\left(\left(q t\left(x_{2}-1\right)+1\right)\left(1+q\left(x_{2}-1\right)\right)^{k-t}-1\right)} \\
& =\sum_{m \geq 0} \frac{1}{\left(x_{2}-1\right)^{m}}\left(\left(q t\left(x_{2}-1\right)+1\right)\left(1+q\left(x_{2}-1\right)\right)^{k-t}-1\right)^{m} \\
& =\sum_{m \geq 0} \frac{1}{\left(x_{2}-1\right)^{m}} \sum_{a=0}^{m}\left(\begin{array}{c}
m \\
a
\end{array}\right)(-1)^{m-a}\left(q t\left(x_{2}-1\right)+1\right)^{a}\left(1+q\left(x_{2}-1\right)\right)^{(k-t) a} \\
& =\sum_{m \geq 0} \frac{1}{\left(x_{2}-1\right)^{m}} \sum_{a=0}^{m} \sum_{b=0}^{a} \sum_{c=0}^{(k-t) a}(-1)^{m-a}\left(\begin{array}{c}
m \\
a
\end{array}\right)\left(\begin{array}{c}
a \\
b
\end{array}\right)\left(\begin{array}{c}
(k-t) a \\
c
\end{array}\right) q^{b} t^{b}\left(x_{2}-1\right)^{b} q^{c}\left(x_{2}-1\right)^{c} .
\end{aligned}
$$

Again, if we want to take the coefficient of $q^{n}$, then we must have $b+c=n$ or $c=n-b$. Thus

$$
A_{k}=\sum_{n \geq 0} q^{n} \sum_{m \geq 0} \sum_{a=0}^{m} \sum_{b=0}^{a}(-1)^{m-a}\left(\begin{array}{c}
m \\
a
\end{array}\right)\left(\begin{array}{l}
a \\
b
\end{array}\right)\left(\begin{array}{c}
(k-t) a \\
n-b
\end{array}\right) t^{b}\left(x_{2}-1\right)^{n} .
$$

Taking the coefficient of $q^{n}$ of both sides of [4.9, we see that

$$
\sum_{\pi \in[k]^{n}} x_{2}^{\overleftarrow{\operatorname{des}}_{\{t+1, \ldots k\}}(\pi)}=\sum_{m \geq 0} \sum_{a=0}^{m} \sum_{b=0}^{a}(-1)^{m-a}\left(\begin{array}{l}
m \\
a
\end{array}\right)\left(\begin{array}{l}
a \\
b
\end{array}\right)\left(\begin{array}{c}
(k-t) a \\
n-b
\end{array}\right) t^{b}\left(x_{2}-1\right)^{n-m}
$$

for all $n$. However, if we replace $x_{2}$ by $z+1$ in (4.3), we see that the polynomial

$$
\sum_{\pi \in[k]^{n}}(z+1)^{\overleftarrow{\operatorname{des}}_{\{t+1, \ldots, k\}}(\pi)}
$$

has the Laurent expansion

$$
\sum_{m \geq 0} \sum_{a=0}^{m} \sum_{b=0}^{a}(-1)^{m-a}\left(\begin{array}{c}
m \\
a
\end{array}\right)\left(\begin{array}{l}
a \\
b
\end{array}\right)\left(\begin{array}{c}
(k-t) a \\
n-b
\end{array}\right) t^{b} z^{n-m}
$$

It follows that it must be the case that

$$
\sum_{m \geq n+1} \sum_{a=0}^{m} \sum_{b=0}^{a}(-1)^{m-a}\left(\begin{array}{c}
m \\
a
\end{array}\right)\left(\begin{array}{l}
a \\
b
\end{array}\right)\left(\begin{array}{c}
(k-t) a \\
n-b
\end{array}\right) t^{b} z^{n-m}=0,
$$


so that

$$
A_{k}=\sum_{n \geq 0} q^{n} \sum_{m=0}^{n} \sum_{a=0}^{m} \sum_{b=0}^{a}(-1)^{m-a}\left(\begin{array}{l}
m \\
a
\end{array}\right)\left(\begin{array}{l}
a \\
b
\end{array}\right)\left(\begin{array}{c}
(k-t) a \\
n-b
\end{array}\right) t^{b}\left(x_{1}-1\right)^{n-m}
$$

Thus if we take the coefficient of $x_{2}^{s}$ on both sides of (4.11) and we use the remark in the introduction that $\overleftarrow{\operatorname{des}}_{\{t+1, \ldots, k\}}(w)=\overleftarrow{\operatorname{ris}}_{[k-t]}(w)$ for all $w \in[k]^{n}$, then we have that the number of words $w \in[k]^{n}$ such that $\overleftarrow{\operatorname{des}}_{\{t+1, \ldots, k\}}(w)=s\left(\overleftarrow{\operatorname{ris}}_{[k-t]}(w)=s\right)$ is equal to

$$
\sum_{m=0}^{n-s} \sum_{a=0}^{m} \sum_{b=0}^{a}(-1)^{n-a-s}\left(\begin{array}{l}
m \\
a
\end{array}\right)\left(\begin{array}{l}
a \\
b
\end{array}\right)\left(\begin{array}{c}
(k-t) a \\
n-b
\end{array}\right)\left(\begin{array}{c}
n-m \\
s
\end{array}\right) t^{b} .
$$

Reordering the summands, we see that 4.12 is equal to

$$
\sum_{b=0}^{n-s} t^{b} \sum_{a=b}^{n-s}(-1)^{n-a-s}\left(\begin{array}{l}
a \\
b
\end{array}\right)\left(\begin{array}{c}
(k-t) a \\
n-b
\end{array}\right) \sum_{m=a}^{n-s}\left(\begin{array}{c}
m \\
a
\end{array}\right)\left(\begin{array}{c}
n-m \\
s
\end{array}\right) .
$$

However it is easy to see that

$$
\sum_{m=a}^{n-s}\left(\begin{array}{c}
m \\
a
\end{array}\right)\left(\begin{array}{c}
n-m \\
s
\end{array}\right)=\left(\begin{array}{c}
n+1 \\
a+1+s
\end{array}\right) .
$$

because the LHS of (4.14) is the result of classifying the ways to pick $a+1+s$ points from $[n+1]$ by the value $m+1$ of the $(a+1)$-st point reading from left to right. Thus we have obtain the following theorem.

Theorem 4.2 If $\leq k$, then the number of words $w \in[k]^{n}$ such that $\overleftarrow{\operatorname{des}}_{\{t+1, \ldots, k\}}(w)=s\left(\overleftarrow{\operatorname{ris}}_{[k-t]}(w)=\right.$ s) is equal to

$$
\sum_{b=0}^{n-s} t^{b} \sum_{a=b}^{n-s}(-1)^{n-a-s}\left(\begin{array}{l}
a \\
b
\end{array}\right)\left(\begin{array}{c}
(k-t) a \\
n-b
\end{array}\right)\left(\begin{array}{c}
n+1 \\
a+s+1
\end{array}\right)
$$

In some special cases, we can give direct combinatorial proofs of Theorems 4.1 and 4.2. For example, in the special case of Theorem 4.2 where $t=k-1$, one can use the Pfaff-Saalschütz Theorem to show that

$$
\left(\begin{array}{l}
b \\
s
\end{array}\right)\left(\begin{array}{c}
n-b \\
s
\end{array}\right)=\sum_{a=b}^{n-s}(-1)^{n-s-a}\left(\begin{array}{l}
a \\
b
\end{array}\right)\left(\begin{array}{c}
a \\
n-b
\end{array}\right)\left(\begin{array}{c}
n+1 \\
a+s+1
\end{array}\right) .
$$

so that 4.15 reduces to

$$
\sum_{b=0}^{n-s}(k-1)^{b}\left(\begin{array}{l}
b \\
s
\end{array}\right)\left(\begin{array}{c}
n-b \\
s
\end{array}\right) .
$$

It is easy to see that 4.17 is the result of classifying the words such that $\overleftarrow{\operatorname{des}}_{\{k\}}(w)=s$ by the number of letters which are not equal to $k$ in the word. That is, if there are $b$ such letters, then we can pick a word of length $b$ in the alphabet $\{1, \ldots, k-1\}$ in $(k-1)^{b}$ ways. Next we insert a $k$ directly in front of $s$ different letters in $u$ in $\left(\begin{array}{l}b \\ s\end{array}\right)$ ways to create $s$ descents that start with $k$. Finally we can place the remaining $n-b-s$ $k$ 's either in a block with one of the $k$ 's that start a descent or at the end of $u$. The number of ways to place the remaining $k$ 's is the number of non-negative integer valued solutions to $x_{1}+\cdots+x_{s+1}=n-b-s$ or, equivalently, the number of positive integer valued solutions to $y_{1}+\cdots+y_{s+1}=n-b+1$ which is clearly $\left(\begin{array}{c}n-b \\ s\end{array}\right)$. We can give a similar combinatorial proof of Theorem 4.1 in the special case when $t=2$. 


\section{Classifying descents and rises by their equivalence classes $\bmod s$ for $s \geq 2$.}

In this section we study the set partition $\mathbb{N}=\mathbb{N}_{1} \cup \mathbb{N}_{2} \cup \cdots \cup \mathbb{N}_{s}$ where $s>2$ and $\mathbb{N}_{i}=\{j \mid j=i$ $\bmod s\}$ for $i=1, \ldots, s$. In this case, we shall denote $N_{i}=s \mathbb{N}+i$ for $i=1, \ldots, s-1$ and $N_{s}=s \mathbb{N}$.

Recall that we can rewrite 2.7) as

$$
A_{k}=\frac{1+\sum_{j=1}^{k} \lambda_{j} \prod_{i=j+1}^{k} \mu_{i}}{1-\sum_{j=1}^{k} \nu_{j} \prod_{i=j+1}^{k} \mu_{i}}
$$

where $\lambda_{j}=\frac{q_{j}\left(1-y_{j}\right)}{1-q_{j}\left(z_{j}-y_{j}\right)}, \mu_{i}=\frac{1-q_{i}\left(z_{i}-x_{i}\right)}{1-q_{i}\left(z_{i}-y_{i}\right)}$, and $\nu_{j}=\frac{q_{j} y_{j}}{1-q_{j}\left(z_{j}-y_{j}\right)}$.

We let $A_{k}^{(s)}$ denote $A_{k}$ under the substitution $\lambda_{s i+j}=\lambda_{j}, \mu_{s i+j}=\mu_{j}$, and $\nu_{s i+j}=\nu_{j}$ for all $i$ and $j=1, \ldots, s$. Then it is easy to see that for $k \geq 1$,

$$
\begin{aligned}
A_{s k}^{(s)} & =\frac{1+\left(\sum_{j=1}^{s} \lambda_{j} \prod_{i=j+1}^{s} \mu_{i}\right)\left(\sum_{r=0}^{k-1}\left(\mu_{1} \mu_{2} \cdots \mu_{s}\right)^{r}\right)}{1-\left(\sum_{j=1}^{s} \nu_{j} \prod_{i=j+1}^{s} \mu_{i}\right)\left(\sum_{r=0}^{k-1}\left(\mu_{1} \mu_{2} \cdots \mu_{s}\right)^{r}\right)} \\
& =\frac{1+\left(\sum_{j=1}^{s} \lambda_{j} \prod_{i=j+1}^{s} \mu_{i}\right)\left(\frac{\left(\mu_{1} \mu_{2} \cdots \mu_{s}\right)^{k}-1}{\left(\mu_{1} \mu_{2} \cdots \mu_{s}\right)-1}\right)}{1-\left(\sum_{j=1}^{s} \nu_{j} \prod_{i=j+1}^{s} \mu_{i}\right)\left(\frac{\left(\mu_{1} \mu_{2} \cdots \mu_{s}\right)^{k}-1}{\left(\mu_{1} \mu_{2} \cdots \mu_{s}\right)-1}\right)} .
\end{aligned}
$$

Similarly for $1 \leq t \leq s-1$,

$$
\begin{aligned}
& A_{s k+t}^{(s)}= \\
& \frac{1+\left(\sum_{j=1}^{t} \lambda_{j} \prod_{i=j+1}^{t} \mu_{i}\right)\left(\sum_{r=0}^{k}\left(\mu_{1} \cdots \mu_{s}\right)^{r}\right)+\left(\mu_{1} \mu_{2} \cdots \mu_{t}\right)\left(\sum_{j=t+1}^{s} \lambda_{j} \prod_{i=j+1}^{s} \mu_{i}\right)\left(\sum_{r=0}^{k-1}\left(\mu_{1} \cdots \mu_{s}\right)^{r}\right)}{1-\left(\sum_{j=1}^{t} \nu_{j} \prod_{i=j+1}^{t} \mu_{i}\right)\left(\sum_{r=0}^{k}\left(\mu_{1} \cdots \mu_{s}\right)^{r}\right)-\left(\mu_{1} \mu_{2} \cdots \mu_{t}\right)\left(\sum_{j=t+1}^{s} \nu_{j} \prod_{i=j+1}^{s} \mu_{i}\right)\left(\sum_{r=0}^{k-1}\left(\mu_{1} \cdots \mu_{s}\right)^{r}\right)} \\
& \frac{1+\left(\sum_{j=1}^{t} \lambda_{j} \prod_{i=j+1}^{t} \mu_{i}\right)\left(\frac{\left(\mu_{1} \mu_{2} \cdots \mu_{s}\right)^{k+1}-1}{\left(\mu_{1} \mu_{2} \cdots \mu_{s}\right)-1}\right)+\left(\mu_{1} \mu_{2} \cdots \mu_{t}\right)\left(\sum_{j=t+1}^{s} \lambda_{j} \prod_{i=j+1}^{s} \mu_{i}\right)\left(\frac{\left(\mu_{1} \mu_{2} \cdots \mu_{s}\right)^{k}-1}{\left(\mu_{1} \mu_{2} \cdots \mu_{s}\right)-1}\right)}{1-\left(\sum_{j=1}^{t} \nu_{j} \prod_{i=j+1}^{t} \mu_{i}\right)\left(\frac{\left(\mu_{1} \mu_{2} \cdots \mu_{s}\right)^{k+1}-1}{\left(\mu_{1} \mu_{2} \cdots \mu_{s}\right)-1}\right)-\left(\mu_{1} \mu_{2} \cdots \mu_{t}\right)\left(\sum_{j=t+1}^{s} \nu_{j} \prod_{i=j+1}^{s} \mu_{i}\right)\left(\frac{\left(\mu_{1} \mu_{2} \cdots \mu_{s}\right)^{k}-1}{\left(\mu_{1} \mu_{2} \cdots \mu_{s}\right)-1}\right)}
\end{aligned}
$$

\subsection{The case where $k$ is equal to 0 mod $s$.}

First we shall consider formulas for the number of words in $[s k]^{n}$ with $p$ descents whose first element is congruent to $r \bmod s$ where $1 \leq r \leq s$. Note that if we consider the complement map comp $p_{s k}$ : $[s k]^{n} \rightarrow[s k]^{n}$ given by $\operatorname{comp}\left(\pi_{1} \cdots \pi_{n}\right)=\left(s k+1-\pi_{1}\right) \cdots\left(s k+1-\pi_{n}\right)$, then it is easy to see that $\overleftarrow{\operatorname{des}}_{s \mathbb{N}+r}(\pi)=\overleftarrow{\operatorname{ris}}_{s \mathbb{N}+s+1-r}\left(\operatorname{comp}_{s k}(\pi)\right)$ for $r=1, \ldots, s$. Thus the problem of counting the number of words in $[s k]^{n}$ with $p$ descents whose first element is congruent to $r \bmod s$ is the same as counting the number of words in $[s k]^{n}$ with $p$ rises whose first element is congruent to $s+1-r \bmod s$

Now consider the case where $z_{i}=y_{i}=1$ and $q_{i}=q$ for $i=1, \ldots, s$ and $x_{i}=1$ for $i \neq r$. In this case,

$$
A_{s k}^{(s)}=\sum_{n \geq 0} q^{n} \sum_{\pi \in[s k]^{n}} x_{r}^{\overleftarrow{\mathrm{des}}_{s \mathbb{N}+r}(\pi)}
$$


Substituting into our formulas for $A_{s k}^{(s)}$, we see that in this case $\lambda_{i}=0$ and $\nu_{i}=q$ for $i=1, \ldots, s$ and $\mu_{i}=1$ for $i \neq r$ and $\mu_{r}=1+q\left(x_{r}-1\right)$. Thus under this substitution, 5.2p becomes

$$
\begin{aligned}
A_{s k}^{(s)} & =\frac{1}{1-\left((r-1) q \mu_{r}+(s-r+1) q\right) \frac{\mu_{r}^{k}-1}{q\left(x_{r}-1\right)}} \\
& =\frac{1}{1-\frac{1}{\left(x_{r}-1\right)}\left(s+(r-1) q\left(x_{r}-1\right)\right)\left(\mu_{r}^{k}-1\right)} \\
& =\sum_{j=0}^{\infty} \frac{1}{\left(x_{r}-1\right)^{j}}\left(s+(r-1) q\left(x_{r}-1\right)\right)^{j}\left(\mu_{r}^{k}-1\right)^{j} \\
& =\sum_{j=0}^{\infty} \frac{1}{\left(x_{r}-1\right)^{j}} \sum_{i_{1}, i_{2}=0}^{j}\left(\begin{array}{c}
j \\
i_{1}
\end{array}\right) s^{j-i_{1}}(r-1)^{i_{1}} q^{i_{1}}\left(x_{r}-1\right)^{i_{1}}\left(\begin{array}{c}
j \\
i_{2}
\end{array}\right)(-1)^{j-i_{2}} \mu_{r}^{k i_{2}} \\
& =\sum_{j=0}^{\infty} \frac{1}{\left(x_{r}-1\right)^{j}} \sum_{i_{1}, i_{2}=0}^{j}\left(\begin{array}{c}
j \\
i_{1}
\end{array}\right) s^{j-i_{1}}(r-1)^{i_{1}} q^{i_{1}}\left(x_{r}-1\right)^{i_{1}}\left(\begin{array}{c}
j \\
i_{2}
\end{array}\right)(-1)^{j-i_{2}}\left(\sum_{t=0}^{k i_{2}}\left(\begin{array}{c}
k i_{2} \\
t
\end{array}\right) q^{t}\left(x_{r}-1\right)^{t}\right) .
\end{aligned}
$$

Taking the coefficient of $q^{n}$ in (5.3), we see that $n=t+i_{1}$ so that

$$
A_{s k}^{(s)}=\sum_{n \geq 0} q^{n} \sum_{j=0}^{\infty} \sum_{i_{1}, i_{2}=0}^{j}(-1)^{j-i_{2}} s^{j-i_{1}}(r-1)^{i_{1}}\left(\begin{array}{c}
j \\
i_{1}
\end{array}\right)\left(\begin{array}{c}
j \\
i_{2}
\end{array}\right)\left(\begin{array}{c}
k i_{2} \\
n-i_{1}
\end{array}\right)\left(x_{r}-1\right)^{n-j} .
$$

Thus we must have

$$
\sum_{\pi \in[s k]^{n}} x_{r}^{\overleftarrow{\operatorname{des}}_{s \mathrm{~N}+r}(\pi)}=\sum_{j=0}^{\infty} \sum_{i_{1}, i_{2}=0}^{j}(-1)^{j-i_{2}} s^{j-i_{1}}(r-1)^{i_{1}}\left(\begin{array}{c}
j \\
i_{1}
\end{array}\right)\left(\begin{array}{c}
j \\
i_{2}
\end{array}\right)\left(\begin{array}{c}
k i_{2} \\
n-i_{1}
\end{array}\right)\left(x_{r}-1\right)^{n-j}
$$

for all $n$. However, if we replace $x_{r}$ by $z+1$ in 5.4, we see that the polynomial

$$
\sum_{\pi \in[s k]^{n}}(z+1)^{\overleftarrow{\operatorname{des}}_{s \mathbb{N}+r}(\pi)}
$$

has the Laurent expansion

$$
\sum_{j=0}^{\infty} \sum_{i_{1}, i_{2}=0}^{j}(-1)^{j-i_{2}} s^{j-i_{1}}(r-1)^{i_{1}}\left(\begin{array}{c}
j \\
i_{1}
\end{array}\right)\left(\begin{array}{c}
j \\
i_{2}
\end{array}\right)\left(\begin{array}{c}
k i_{2} \\
n-i_{1}
\end{array}\right) z^{n-j}
$$

It follows that it must be the case that

$$
\sum_{j=n+1}^{\infty} \sum_{i_{1}, i_{2}=0}^{j}(-1)^{j-i_{2}} s^{j-i_{1}}(r-1)^{i_{1}}\left(\begin{array}{c}
j \\
i_{1}
\end{array}\right)\left(\begin{array}{c}
j \\
i_{2}
\end{array}\right)\left(\begin{array}{c}
k i_{2} \\
n-i_{1}
\end{array}\right) z^{n-j}=0
$$

so that

$$
A_{s k}^{(s)}=\sum_{n \geq 0} q^{n} \sum_{j=0}^{n} \sum_{i_{1}, i_{2}=0}^{j}(-1)^{j-i_{2}} s^{j-i_{1}}(r-1)^{i_{1}}\left(\begin{array}{c}
j \\
i_{1}
\end{array}\right)\left(\begin{array}{c}
j \\
i_{2}
\end{array}\right)\left(\begin{array}{c}
k i_{2} \\
n-i_{1}
\end{array}\right)\left(x_{r}-1\right)^{n-j} .
$$

Thus we have the following theorem by taking the coefficient of $x_{r}^{p}$ on both sides of 5.5. 
Theorem 5.1 The number of words $\pi \in[s k]^{n}$ with $\overleftarrow{\operatorname{des}}_{s \mathbb{N}+r}(\pi)=p\left(\overleftarrow{\mathrm{ris}}_{s \mathbb{N}+s+1-r}(\pi)=p\right)$ is

$$
\sum_{j=0}^{n-p} \sum_{i_{1}, i_{2}=0}^{j}(-1)^{n+p+i_{2}} s^{j-i_{1}}(r-1)^{i_{1}}\left(\begin{array}{c}
j \\
i_{1}
\end{array}\right)\left(\begin{array}{c}
j \\
i_{2}
\end{array}\right)\left(\begin{array}{c}
k i_{2} \\
n-i_{1}
\end{array}\right)\left(\begin{array}{c}
n-j \\
p
\end{array}\right) .
$$

In the case $s=2$, our formulas simplify somewhat. For example, putting $s=2$ and $r=2$ in Theorem 5.1 we obtain the following.

Corollary 5.2 The number of n-letter words $\pi$ on $[2 k]$ having $\overleftarrow{\operatorname{des}}_{E}(\pi)=p$ (resp. $\overleftarrow{\operatorname{ris}}_{O}(\pi)=p$ ) is given by

$$
\sum_{j=0}^{n-s} \sum_{i_{1}, i_{2}=0}^{j}(-1)^{n+p+i_{2}} 2^{j-i_{1}}\left(\begin{array}{c}
j \\
i_{1}
\end{array}\right)\left(\begin{array}{c}
j \\
i_{2}
\end{array}\right)\left(\begin{array}{c}
k i_{2} \\
n-i_{1}
\end{array}\right)\left(\begin{array}{c}
n-j \\
p
\end{array}\right) .
$$

Similarly, putting $s=2$ and $r=1$ in Theorem 5.1, we obtain the following.

Corollary 5.3 The number of n-letter words $\pi$ on $[2 k]$ having $\overleftarrow{\operatorname{des}}_{O}(\pi)=p$ (resp. $\overleftarrow{\text { ris }}_{E}(\pi)=p$ ) is given by

$$
\sum_{j=0}^{n-p} \sum_{i=0}^{j}(-1)^{n+p+i} 2^{j}\left(\begin{array}{c}
j \\
i
\end{array}\right)\left(\begin{array}{c}
k i \\
n
\end{array}\right)\left(\begin{array}{c}
n-j \\
p
\end{array}\right)
$$

\subsection{The cases where $k$ is equal to $t \bmod s$ for $t=1, \ldots, s-1$.}

Fix $t$ where $1 \leq t \leq s-1$. First we shall consider formulas for the number of words in $[s k+t]^{n}$ with $p$ descents whose first element is congruent to $r \bmod s$ where $1 \leq r \leq s$. We shall see that we have to divide this problem into two cases depending on whether $r \leq t$ or $r>t$. Note that if we consider the complement map comp $p_{s k+t}:[s k+t]^{n} \rightarrow[s k+t]^{n}$ given by $\operatorname{comp}\left(\pi_{1} \cdots \pi_{n}\right)=$ $\left(s k+t+1-\pi_{1}\right) \cdots\left(s k+t+1-\pi_{n}\right)$, then it is easy to see that $\overleftarrow{\operatorname{des}}_{s \mathbb{N}+r}(\pi)=\overleftarrow{\operatorname{ris}}_{s \mathbb{N}+t+1-r}\left(\operatorname{comp}_{s k}(\pi)\right)$ for $r=1, \ldots, t$ and $\overleftarrow{\operatorname{des}}_{s \mathbb{N}+r}(\pi)=\overleftarrow{\text { ris }}_{s \mathbb{N}+s+r-t-1}\left(\operatorname{comp}_{s k}(\pi)\right)$ for $r=t+1, \ldots, s$

First consider the case where $y_{i}=z_{i}=1$ for $i=1, \ldots, s$ and $x_{i}=1$ for $i \neq r$ where $r>t$. In this case,

$$
A_{s k+t}^{(s)}=\sum_{n \geq 0} q^{n} \sum_{\pi \in[s k+t]^{n}} x_{r}^{\overleftarrow{\mathrm{des}}_{s \mathbb{N}+r}(\pi)}
$$

Substituting into our formulas for $A_{s k+t}^{(s)}$, we see that in this case $\lambda_{i}=0$ and $\nu_{i}=q$ for $i=1, \ldots, s$ and 
$\mu_{i}=1$ for $i \neq r$ and $\mu_{r}=1+q\left(x_{r}-1\right)$. Thus under this substitution, 5.2 becomes

$$
\begin{aligned}
A_{s k+t}^{(s)} & =\frac{1}{1-q t \frac{\mu_{r}^{k+1}-1}{q\left(x_{r}-1\right)}-\left((r-1-t) q \mu_{r}+(s-r+1) q\right) \frac{\mu_{r}^{k}-1}{q\left(x_{r}-1\right)}} \\
& =\frac{1}{1-\frac{1}{\left(x_{r}-1\right)}\left[t\left(\mu_{r}^{k+1}-1\right)+\left(s-t+(r-1-t) q\left(x_{r}-1\right)\right)\left(\mu_{r}^{k}-1\right)\right]} \\
& =\frac{1}{1-\frac{1}{\left(x_{r}-1\right)}\left[\mu_{r}^{k}\left[s+(r-1) q\left(x_{r}-1\right)\right]-\left[s+(r-1-t) q\left(x_{r}-1\right)\right]\right]} \\
& =\sum_{m=0}^{\infty} \frac{1}{\left(x_{r}-1\right)^{m}}\left[\mu_{r}^{k}\left[s+(r-1) q\left(x_{r}-1\right)\right]-\left[s+(r-1-t) q\left(x_{r}-1\right)\right]\right]^{m} \\
& =\sum_{m=0}^{\infty} \sum_{j=0}^{m} \frac{(-1)^{m-j}}{\left(x_{r}-1\right)^{m}}\left(\begin{array}{c}
m \\
j
\end{array}\right)\left(s+(r-1-t) q\left(x_{r}-1\right)\right)^{m-j}\left(s+(r-1) q\left(x_{r}-1\right)\right)^{j} \mu_{r}^{k j} .
\end{aligned}
$$

Using the expansions

$$
\begin{aligned}
\left(s+(r-1-t) q\left(x_{r}-1\right)\right)^{m-j} & =\sum_{i_{1}=0}^{m-j}\left(\begin{array}{c}
m-j \\
i_{1}
\end{array}\right) s^{m-j-i_{1}}(r-1-t)^{i_{1}} q^{i_{1}}\left(x_{r}-1\right)^{i_{1}}, \\
\left(s+(r-1) q\left(x_{r}-1\right)\right)^{j} & =\sum_{i_{2}=0}^{j}\left(\begin{array}{c}
j \\
i_{2}
\end{array}\right) s^{j-i_{2}}(r-1)^{i_{2}} q^{i_{2}}\left(x_{r}-1\right)^{i_{2}}, \text { and } \\
\mu_{r}^{k j} & =\sum_{i_{3}=0}^{k j}\left(\begin{array}{c}
k j \\
i_{3}
\end{array}\right) q^{i_{3}}\left(x_{r}-1\right)^{i_{3}},
\end{aligned}
$$

and setting $i_{1}+i_{2}+i_{3}=n$, we see that 5.7 becomes

$$
\begin{aligned}
& A_{s k+t}^{(s)}= \\
& \sum_{n \geq 0} q^{n} \sum_{m=0}^{\infty} \sum_{j=0}^{m} \sum_{i_{1}=0}^{m-j} \sum_{i_{2}=0}^{j}(-1)^{m-j} s^{m-i_{1}-i_{2}}(r-1-t)^{i_{1}}(r-1)^{i_{2}} \times \\
& \quad\left(\begin{array}{c}
m \\
j
\end{array}\right)\left(\begin{array}{c}
m-j \\
i_{1}
\end{array}\right)\left(\begin{array}{c}
j \\
i_{2}
\end{array}\right)\left(\begin{array}{c}
k j \\
n-i_{1}-i_{2}
\end{array}\right)\left(x_{r}-1\right)^{n-m} .
\end{aligned}
$$

Thus we must have

$$
\begin{aligned}
& \sum_{\pi \in\left[s k_{t}\right]^{n}} x_{r}^{\overleftarrow{\operatorname{des}_{s \mathbb{N}+r}}(\pi)}= \\
& \sum_{m=0}^{\infty} \sum_{j=0}^{m} \sum_{i_{1}=0}^{m-j} \sum_{i_{2}=0}^{j}(-1)^{m-j} s^{m-i_{1}-i_{2}}(r-1-t)^{i_{1}}(r-1)^{i_{2}} \times \\
& \quad\left(\begin{array}{c}
m \\
j
\end{array}\right)\left(\begin{array}{c}
m-j \\
i_{1}
\end{array}\right)\left(\begin{array}{c}
j \\
i_{2}
\end{array}\right)\left(\begin{array}{c}
k j \\
n-i_{1}-i_{2}
\end{array}\right)\left(x_{r}-1\right)^{n-m}
\end{aligned}
$$


for all $n$. However, if we replace $x_{r}$ by $z+1$ in (5.8), we see that the polynomial

$$
\sum_{\pi \in[s k+t]^{n}}(z+1)^{\overleftarrow{\operatorname{des}}_{s \mathbb{N}+r}(\pi)}
$$

has the Laurent expansion

$$
\sum_{m=0}^{\infty} \sum_{j=0}^{m} \sum_{i_{1}=0}^{m-j} \sum_{i_{2}=0}^{j}(-1)^{m-j} s^{m-i_{1}-i_{2}}(r-1-t)^{i_{1}}(r-1)^{i_{2}}\left(\begin{array}{c}
m \\
j
\end{array}\right)\left(\begin{array}{c}
m-j \\
i_{1}
\end{array}\right)\left(\begin{array}{c}
j \\
i_{2}
\end{array}\right)\left(\begin{array}{c}
k j \\
n-i_{1}-i_{2}
\end{array}\right) z^{n-m}
$$

It follows that

$$
\sum_{m=n+1}^{\infty} \sum_{j=0}^{m} \sum_{i_{1}=0}^{m-j} \sum_{i_{2}=0}^{j}(-1)^{m}-j s^{m-i_{1}-i_{2}}(r-1-t)^{i_{1}}(r-1)^{i_{2}}\left(\begin{array}{c}
m \\
j
\end{array}\right)\left(\begin{array}{c}
m-j \\
i_{1}
\end{array}\right)\left(\begin{array}{c}
j \\
i_{2}
\end{array}\right)\left(\begin{array}{c}
k j \\
n-i_{1}-i_{2}
\end{array}\right) z^{n-m}=0
$$

so that

$$
\begin{aligned}
& A_{s k+t}^{(s)}= \\
& \sum_{m=0}^{n} \sum_{j=0}^{m} \sum_{i_{1}=0}^{m-j} \sum_{i_{2}=0}^{j}(-1)^{m-j} s^{m-i_{1}-i_{2}}(r-1-t)^{i_{1}}(r-1)^{i_{2}}\left(\begin{array}{c}
m \\
j
\end{array}\right)\left(\begin{array}{c}
m-j \\
i_{1}
\end{array}\right)\left(\begin{array}{c}
j \\
i_{2}
\end{array}\right)\left(\begin{array}{c}
k j \\
n-i_{1}-i_{2}
\end{array}\right)\left(x_{r}-1\right)^{n-m} .
\end{aligned}
$$

Thus we have the following theorem by taking the coefficient of $x_{r}^{p}$ on both sides of [5.9].

Theorem 5.4 If $t=1, \ldots, s-1$ and $t<r \leq s$, then the number of words $\pi \in[s k+t]^{n}$ with $\overleftarrow{\operatorname{des}}_{s \mathbb{N}+r}(\pi)=p\left(\overleftarrow{\operatorname{ris}}_{s \mathbb{N}+s+r-t-1}(\pi)=p\right)$ is

$$
\sum_{m=0}^{n-p} \sum_{j=0}^{m} \sum_{i_{1}=0}^{m-j} \sum_{i_{2}=0}^{j}(-1)^{n+p+j} s^{m-i_{1}-i_{2}}(r-1-t)^{i_{1}}(r-1)^{i_{2}}\left(\begin{array}{c}
m \\
j
\end{array}\right)\left(\begin{array}{c}
m-j \\
i_{1}
\end{array}\right)\left(\begin{array}{c}
j \\
i_{2}
\end{array}\right)\left(\begin{array}{c}
k j \\
n-i_{1}-i_{2}
\end{array}\right)\left(\begin{array}{c}
n-m \\
p
\end{array}\right) .
$$

In the case $s=2$, our formulas simplify somewhat. For example, putting $s=2, r=2$ and $t=1$ in Theorem 5.4 we obtain the following.

Corollary 5.5 The number of n-letter words $\pi$ over $[2 k+1]$ having $\overleftarrow{\operatorname{des}}_{E}(\pi)=p\left(\right.$ resp. $\left.\overleftarrow{\operatorname{ris}}_{E}(\pi)=p\right)$ is given by

$$
\sum_{m=0}^{n-p} \sum_{j=0}^{m} \sum_{i=0}^{j}(-1)^{n+p+j} 2^{m-i}\left(\begin{array}{c}
m \\
j
\end{array}\right)\left(\begin{array}{c}
j \\
i
\end{array}\right)\left(\begin{array}{c}
k j \\
n-i
\end{array}\right)\left(\begin{array}{c}
n-m \\
p
\end{array}\right) .
$$

Next consider the case where $y_{i}=z_{i}=1$ for $i=1, \ldots, s$ and $x_{i}=1$ for $i \neq r$ where $r \leq t$. In this case,

$$
A_{s k+t}^{(s)}=\sum_{n \geq 0} q^{n} \sum_{\pi \in[s k+t]^{n}} x_{r}^{\overleftarrow{\operatorname{des}}_{s \mathbb{N}+r}(\pi)}
$$


Substituting into our formulas for $A_{s k+t}^{(s)}$, we see that in this case $\lambda_{i}=0$ and $\nu_{i}=q$ for $i=1, \ldots, s$ and $\mu_{i}=1$ for $i \neq r$ and $\mu_{r}=1+q\left(x_{r}-1\right)$. Thus, under this substitution, 5.2 becomes

$$
\begin{aligned}
A_{s k+t}^{(s)} & =\frac{1}{\left.1-\left((r-1) q \mu_{r}\right)+q(t-r+1)\right) \frac{\mu_{r}^{k+1}-1}{q\left(x_{r}-1\right)}-(s-t) q \mu_{r} \frac{\mu_{r}^{k}-1}{q\left(x_{r}-1\right)}} \\
& =\frac{1}{1-\frac{1}{\left(x_{r}-1\right)}\left[\left(t+(r-1) q\left(x_{r}-1\right)\right)\left(\mu_{r}^{k+1}-1\right)+(s-t) \mu_{r}\left(\mu_{r}^{k}-1\right)\right]} \\
& =\frac{1}{1-\frac{1}{\left(x_{r}-1\right)}\left[\mu_{r}^{k+1}\left[s+(r-1) q\left(x_{r}-1\right)\right]-\left[s+(s-t+r-1) q\left(x_{r}-1\right)\right]\right]} \\
& =\sum_{m=0}^{\infty} \frac{1}{\left(x_{r}-1\right)^{m}}\left[\mu_{r}^{k+1}\left[s+(r-1) q\left(x_{r}-1\right)\right]-\left[s+(s-t+r-1) q\left(x_{r}-1\right)\right]\right]^{m} \\
& =\sum_{m=0}^{\infty} \sum_{j=0}^{m} \frac{(-1)^{m-j}}{\left(x_{r}-1\right)^{m}}\left(\begin{array}{c}
m \\
j
\end{array}\right)\left(s+(s-t+r-1) q\left(x_{r}-1\right)\right)^{m-j}\left(s+(r-1) q\left(x_{r}-1\right)\right)^{j} \mu_{r}^{k j+j}
\end{aligned}
$$

Using the expansions

$$
\begin{aligned}
\left(s+(s-t+r-1) q\left(x_{r}-1\right)\right)^{m-j} & =\sum_{i_{1}=0}^{m-j}\left(\begin{array}{c}
m-j \\
i_{1}
\end{array}\right) s^{m-j-i_{1}}(s-t+r-1)^{i_{1}} q^{i_{1}}\left(x_{r}-1\right)^{i_{1}}, \\
\left(s+(r-1) \mu_{r}^{j}\right. & =\sum_{i_{2}=0}^{j}\left(\begin{array}{c}
j \\
i_{2}
\end{array}\right) s^{j-i_{2}}(r-1)^{i_{2}} q^{i_{2}}\left(x_{r}-1\right)^{i_{2}}, \text { and } \\
\mu_{r}^{k j+j} & =\sum_{i_{3}=0}^{k j+j}\left(\begin{array}{c}
k j+j \\
i_{3}
\end{array}\right) q^{i_{3}}\left(x_{r}-1\right)^{i_{3}},
\end{aligned}
$$

and setting $i_{1}+i_{2}+i_{3}=n$, we see that 5.11 becomes

$$
\begin{gathered}
A_{s k+t}^{(s)}= \\
\sum_{n \geq 0} q^{n} \sum_{m=0}^{\infty} \sum_{j=0}^{m} \sum_{i_{1}=0}^{m-j} \sum_{i_{2}=0}^{j}(-1)^{m-j} s^{m-i_{1}-i_{2}}(s-t+r-1)^{i_{1}}(r-1)^{i_{2}} \times \\
\left(\begin{array}{c}
m \\
j
\end{array}\right)\left(\begin{array}{c}
m-j \\
i_{1}
\end{array}\right)\left(\begin{array}{c}
j \\
i_{2}
\end{array}\right)\left(\begin{array}{c}
k j+j \\
n-i_{1}-i_{2}
\end{array}\right)\left(x_{r}-1\right)^{n-m} .
\end{gathered}
$$

Thus we must have

$$
\begin{gathered}
\sum_{\pi \in\left[s k_{t}\right]^{n}} x_{r}^{\overleftarrow{\mathrm{des}}_{s \mathbb{N}+r}(\pi)}= \\
\sum_{m=0}^{\infty} \sum_{j=0}^{m} \sum_{i_{1}=0}^{m-j} \sum_{i_{2}=0}^{j}(-1)^{m-j} s^{m-i_{1}-i_{2}}(s-t+r-1)^{i_{1}}(r-1)^{i_{2}} \times \\
\left(\begin{array}{c}
m \\
j
\end{array}\right)\left(\begin{array}{c}
m-j \\
i_{1}
\end{array}\right)\left(\begin{array}{c}
j \\
i_{2}
\end{array}\right)\left(\begin{array}{c}
k j+j \\
n-i_{1}-i_{2}
\end{array}\right)\left(x_{r}-1\right)^{n-m} .
\end{gathered}
$$


for all $n$. However, if we replace $x_{r}$ by $z+1$ in $[5.12$, we that the polynomial

$$
\sum_{\pi \in[s k+t]^{n}}(z+1)^{\overleftarrow{\operatorname{des}}_{s \mathbb{N}+r}(\pi)}
$$

has the Laurent expansion

$$
\sum_{m=0}^{\infty} \sum_{j=0}^{m} \sum_{i_{1}=0}^{m-j} \sum_{i_{2}=0}^{j}(-1)^{m}-j s^{m-i_{1}-i_{2}}(s-t+r-1)^{i_{1}}(r-1)^{i_{2}}\left(\begin{array}{c}
m \\
j
\end{array}\right)\left(\begin{array}{c}
m-j \\
i_{1}
\end{array}\right)\left(\begin{array}{c}
j \\
i_{2}
\end{array}\right)\left(\begin{array}{c}
k j+j \\
n-i_{1}-i_{2}
\end{array}\right) z^{n-m} .
$$

It follows that it must be the case that

$$
\sum_{m=n+1}^{\infty} \sum_{j=0}^{m} \sum_{i_{1}=0}^{m-j} \sum_{i_{2}=0}^{j}(-1)^{m-j} s^{m-i_{1}-i_{2}}(s-t+r-1)^{i_{1}}(r-1)^{i_{2}}\left(\begin{array}{c}
m \\
j
\end{array}\right)\left(\begin{array}{c}
m-j \\
i_{1}
\end{array}\right)\left(\begin{array}{c}
j \\
i_{2}
\end{array}\right)\left(\begin{array}{c}
k j+j \\
n-i_{1}-i_{2}
\end{array}\right) z^{n-m}=0
$$

so that

$A_{s k+t}^{(s)}=$

$\sum_{m=0}^{n} \sum_{j=0}^{m} \sum_{i_{1}=0}^{m-j} \sum_{i_{2}=0}^{j}(-1)^{m-j} s^{m-i_{1}-i_{2}}(s-t+r-1)^{i_{1}}(r-1)^{i_{2}}\left(\begin{array}{c}m \\ j\end{array}\right)\left(\begin{array}{c}m-j \\ i_{1}\end{array}\right)\left(\begin{array}{c}j \\ i_{2}\end{array}\right)\left(\begin{array}{c}k j+j \\ n-i_{1}-i_{2}\end{array}\right)\left(x_{r}-1\right)^{n-m}$.

Thus we have the following theorem by taking the coefficient of $x_{r}^{p}$ on both sides of 5.9.

Theorem 5.6 If $k \geq 0, s \geq 2, t=1, \ldots, s-1$, and $t<r \leq s$, then the number of words $\pi \in[s k+t]^{n}$ with $\overleftarrow{\operatorname{des}}_{s \mathbb{N}+r}(\pi)=p\left(\overleftarrow{\mathrm{ris}}_{s \mathbb{N}+s+r-t-1}(\pi)=p\right)$ is

$\sum_{m=0}^{n-p} \sum_{j=0}^{m} \sum_{i_{1}=0}^{m-j} \sum_{i_{2}=0}^{j}(-1)^{n+p+j} s^{m-i_{1}-i_{2}}(s-t+r-1)^{i_{1}}(r-1)^{i_{2}}\left(\begin{array}{c}m \\ j\end{array}\right)\left(\begin{array}{c}m-j \\ i_{1}\end{array}\right)\left(\begin{array}{c}j \\ i_{2}\end{array}\right)\left(\begin{array}{c}k j+j \\ n-i_{1}-i_{2}\end{array}\right)\left(\begin{array}{c}n-m \\ p\end{array}\right)$.

In the case $s=2$, our formulas simplify somewhat. For example, putting $s=2, r=1$ and $t=1$ in Theorem 5.4 we obtain the following.

Corollary 5.7 The number of n-letter words $\pi$ over $[2 k+1]$ having $\overleftarrow{\operatorname{des}}_{O}(\pi)=p\left(\right.$ resp. $\left.\overleftarrow{\operatorname{ris}}_{O}(\pi)=p\right)$ is given by

$$
\sum_{m=0}^{n-p} \sum_{j=0}^{m} \sum_{i=0}^{m-j}(-1)^{n+p+j} 2^{m-i}\left(\begin{array}{c}
m \\
j
\end{array}\right)\left(\begin{array}{c}
m-j \\
i
\end{array}\right)\left(\begin{array}{c}
k j+j \\
n-i
\end{array}\right)\left(\begin{array}{c}
n-m \\
p
\end{array}\right) .
$$

\section{Concluding remarks}

A particular case of the results obtained by Burstein and Mansour in [4] is the distribution of descents (resp. levels, rises), which can be viewed as occurrences of so called generalized patterns 21 (resp. 11, 12 ) in words. To get these distributions from our results, we proceed as follows (we explain only the case of descents; rises and levels can be considered similarly). Set $x_{1}=x_{2}=x, y_{1}=y_{2}=z_{1}=z_{2}=1$, and $q_{1}=q_{2}=q$ in $A_{2 k}^{(2)}$ and $A_{2 k+1}^{(2)}$ to get the distribution in [4, Theorem 2.2] for $\ell=2$ (the case of 
descents/rises). Thus, our results refine and generalize the known distributions of descents, levels, and rises in words.

It is interesting to compare our formulas with formulas of Hall and Remmel [7]. For example, suppose that $X=E$ and $Y=\mathbb{N}$ and $\rho=\left(\rho_{1}, \ldots, \rho_{2 k}\right)$ is a composition of $n$. Then Theorem 1.1 tells that the number of words $\pi$ of $[2 k]^{n}$ such that $\overleftarrow{\operatorname{des}}_{E}(\pi)=p$ is

$$
\left(\begin{array}{c}
a \\
\rho_{2}, \rho_{4}, \ldots, \rho_{2 k}
\end{array}\right) \sum_{r=0}^{p}(-1)^{p-r}\left(\begin{array}{c}
a+r \\
r
\end{array}\right)\left(\begin{array}{c}
n+1 \\
p-r
\end{array}\right) \prod_{i=1}^{k}\left(\begin{array}{c}
\rho_{2 i}+r+\left(\rho_{2 i+1}+\rho_{2 i+3}+\cdots+\rho_{2 k-1}\right) \\
\rho_{2 i}
\end{array}\right)
$$

where $a=\rho_{2}+\rho_{4}+\cdots+\rho_{2 k}$. This shows that once we are given the distribution of the letters for words in $[2 k]^{n}$, we can find an expression for the number of words $\pi$ such that $\overleftarrow{\operatorname{des}}_{E}(\pi)=p$ with a single alternating sum of products of binomial coefficients. This contrasts with Corollary 5.2 where we require a triple alternating sum of products of binomial coefficients to get an expression for the number of words of $[2 k]^{n}$ such that $\overleftarrow{\operatorname{des}}_{E}(\pi)=p$. Of course, we can get a similar expression for the number of words of $[2 k]^{n}$ such that $\overleftarrow{\operatorname{des}}_{E}(\pi)=p$ by summing the formula in $(6.1)$ over all $\left(\begin{array}{c}n+k-1 \\ k-1\end{array}\right)$ compositions of $n$ into $k$ parts but that has the disadvantage of having the outside sum have a large range as $n$ and $k$ get large. Nevertheless, we note that for 6.1) Hall and Remmel have a direct combinatorial proof via a signreversing involution. It is therefore natural to ask whether one can find similar proofs for our formulas in Sections 3 and 4.

There are several ways in which one could extend our research. For example, one can study our refined statistics $\left(\overleftarrow{\operatorname{Des}_{X}}(\pi), \overleftarrow{\operatorname{Ris}_{X}}(\pi), \operatorname{Lev}_{X}(\pi)\right)$ on the set of all words avoiding a fixed pattern or a set of patterns (see [1, 2, 3, 4] for definitions of "patterns in words" and results on them). More generally, instead of considering the set of all words, one can consider a subset of it defined in some way, and then to study the refined statistics on the subset. Also, instead of considering refined descents, levels, and rises (patterns of length 2), one can consider patterns of length 3 and more in which the equivalence class of the first letter is fixed, or, more generally, in which the equivalence classes of more than one letter (possibly all letters) are fixed. Once such a pattern (or set of patterns) is given, the questions on avoidance (or the distribution of occurrences) of the pattern in words over $[k]$ can be raised. 


\section{References}

[1] A. Burstein, Enumeration of words with forbidden patterns, Ph.D. thesis, University of Pennsylvania, 1998.

[2] A. Burstein And T. Mansour, Words restricted by patterns with at most 2 distinct letters, Electron. J. Combin. 9, no. 2, \#R3 (2002).

[3] A. Burstein And T. MAnsour, Words restricted by 3-letter generalized multipermutation patterns, Ann. Combin. 7 (2003), 1-14.

[4] A. Burstein and T. Mansour, Counting occurrences of some subword patterns, Discr. Math. Theor. Comp. Sci. 6 (2003), 001-012.

[5] L. Comtet, Advanced Combinatorics, D. Reidel Publishing Co., Dordrecht, 1974.

[6] J. Hall, J. Liese, AND J. REMMEL, $q$-analogues of formulas counting descent pairs with prescribed tops and bottoms, in preparation.

[7] J. Hall and J. Remmel, Counting descent pairs with prescribed tops and bottoms, J. Combin. Theory, Series A 115, Issue 5, (2008), 693-725.

[8] S. Heubach And T. Mansour, Counting rises, levels, and drops in compositions, Integers: Elect. J. Combin. Number Theory 5 (2005), Article A11.

[9] S. KitaeV And J. Remmel, Classifying descents according to parity, Ann. Combin. 11 (2007), 173-193.

[10] S. Kitaev and J. Remmel, Classifying Descents According to Equivalence mod $k$, Elect. J. Combin. 13(1) (2006), \#R64.

[11] J. LIESE, Classifying ascents and descents with specified equivalences mod $k$, Proceedings of 18th International Conference on Formal Power Series and Algebra Combinatorics, San Diego, CA (2006).

[12] J. LIESE AND J. REMMEL, $q$-analogues of formulas $\mathrm{f}$ or the number of ascents and descents with specified equivalences mod K, Permutation Patterns Conference, 2006.

[13] P. A. Macmahon, Combinatory Analysis, Vol. 1 and 2, Cambridge Univ. Press, Cambridge, 1915 (reprinted by Chelsea, New York, 1955). 